\section{Design and Analysis of Two Low Power SRAM Cell Structures}

\author{
G. Razavipour, A. Afzali-Kusha, and M. Pedram
}

\begin{abstract}
In this paper, two Static Random Access Memory (SRAM) cells that reduce the static power dissipation due to gate and sub-threshold leakage currents are presented. The first cell structure results in reduced gate voltages for the NMOS pass transistors, and thus lowers the gate leakage current. It reduces the subthreshold leakage current by increasing the ground level during the idle (inactive) mode. The second cell structure makes use of PMOS pass transistors to lower the gate leakage current. In addition, dual threshold voltage technology with forward body biasing is utilized with this structure to reduce the subthreshold leakage while maintaining performance. Compared to a conventional SRAM cell, the first cell structure decreases the total gate leakage current by $66 \%$ and the idle power by $58 \%$ and increases the access time by approximately $2 \%$ while the second cell structure reduces the total gate leakage current by $27 \%$ and the idle power by $37 \%$ with no access time degradation.
\end{abstract}

Index Terms- Low-Power, SRAM Cell, Static Power, Gate Leakage, Tunneling Current, Dual Threshold.

\section{INTRODUCTION}

The International Technology Roadmap for Semiconductors predicts the gate equivalent oxide thickness as low as $0.5 \mathrm{~nm}$ for future CMOS technologies [1]. Since the gate leakage current of MOS transistors increases exponentially with the reduction of the oxide thickness over the active region of a transistor, the gate leakage power dissipation is expected to become a significant fraction of the overall chip power dissipation in nanometer CMOS design processes [2]. The gate tunneling current is predicted to increase at a rate of $500 \mathrm{X}$ per technology generation whereas the sub-threshold current increases by only $5 \mathrm{X}[1][3]$. With the dependence of the leakage power on the number of transistors, and given the projected large memory content of future SoC (System on Chip) devices (more than $90 \%$ of the die area by 2014 [4]), it is important to focus on minimizing the leakage power of SRAM structures. There are several sources for the leakage current i.e., the sub-threshold current due to low threshold voltage, the gate leakage due to very thin gate oxides, and band-to-band tunneling leakage due to heavily-doped halo doping profile [5]. Because of the exponential dependency of the gate leakage current on the oxide thickness, this current has the potential to become the dominant factor for future CMOS technologies.

The tunneling current is composed of three major components: (i) gate-to-source and gate-to-drain overlap currents (edge direct tunneling current), (ii) gate-to-channel current (direct tunneling current), part of which goes to the source and the rest flows to the drain, and (iii) gate-tosubstrate current [5]. In bulk CMOS technology, the gate-tosubstrate leakage current is several orders of magnitude lower than the overlap tunneling current and gate-to-channel current. In the ON state, in addition to the overlap tunneling currents, the gate-to-channel tunneling is added to the gate current increasing the total gate tunneling current in this state. There are several techniques for reducing the gate tunneling leakage in digital circuits (see, e.g., [5]-[10]). These techniques reduce the leakage based on the dependencies of the tunneling currents on the terminal voltages, the gate oxide thickness, and the type of the transistor. One of the techniques is to employ PMOS transistors instead of NMOS transistors. In the PMOS transistor, the gate tunneling current is an order of magnitude lower than that of the NMOS transistor in the inversion regime in the same technology [3]. This mainly originates from the higher barrier height $(4.5 \mathrm{eV})$ for the hole tunneling compared to the lower barrier height $(3.1 \mathrm{eV})$ for the electron tunneling [5]. The exponential dependence of the tunneling current on the barrier height and its linear dependence on the transistor width results in a much smaller tunneling current compared to that of NMOS transistor even when the PMOS transistor is made 2-3 times wider than the NMOS transistor. Another method for reducing the gate leakage current minimizes the voltage difference between the gate to the source or the drain terminals [3], [6], and [7].

We present two design techniques that reduce the gate leakage current in the SRAM cells. In both designs, we focus on the static power dissipation in the idle mode where the cell is fully powered on, but no read or write operation is performed. The rest of the paper is organized as follows. In Section II, we briefly review some of the related work. The proposed SRAM cells are described in Section III while simulations results are discussed in Section IV. Finally, summary and conclusions are provided in Section V.

\section{REVIEW OF RELATED WORKS}

In this section, we review some of the previously proposed SRAM cell structures. In [6], an asymmetric SRAM cell design is presented where an NMOS transistor is added to the SRAM cell to reduce the magnitude of the gate voltage when the cell stores ' 0 ' data (is in the zero state.) As a result, compared to a conventional SRAM cell design, the gate leakage decreases in the zero state while it increases in the one state. The penalty is an increase in the SRAM cell area and longer read access and write times. For this cell, the DC noise margin (data storage integrity) is nearly unchanged [6].

Another method for reducing the gate leakage current in the SRAM cell has been suggested in [3]. In this work, the NCSRAM design, whose circuit diagram is shown in

Fig. 1(a), employs dynamic voltage scaling to reduce the leakage power of the SRAM cells while retaining the stored data during the idle mode. The key idea behind NC-SRAM is the use of two pass transistors $\mathrm{NC} 1$ and $\mathrm{NC} 2$ which provide different ground levels to the memory cell in the active and idle modes. The positive voltage (virtual ground) reduces the gate leakage and subthreshold currents of the cell while degrading the read and write performances [3].

Using dual gate oxide thicknesses is another approach for reducing the gate leakage current in the SRAM cell [8]. In this technique, the gate oxide thicknesses of the NMOS pass transistors and the NMOS pull down transistors are increased. Because the much lower gate leakage of PMOS transistor, no 
change is made to the gate oxide thickness of the PMOS pull up transistors. To achieve a lower subthreshold current, the dual threshold voltage technique has been used. The cell evaluation is performed by using the high threshold voltage for different transistors. In the best case, the power consumption is decreased and the stability is improved but the read access time is degraded [8]. In [9], a low power ninetransistor SRAM cell structure has been proposed. In this structure, to improve the stability in the read mode, three NMOS transistors are added to the cell to separate the read and write circuits. Indeed, the cell stability is improved at the cost of increasing the cell area.

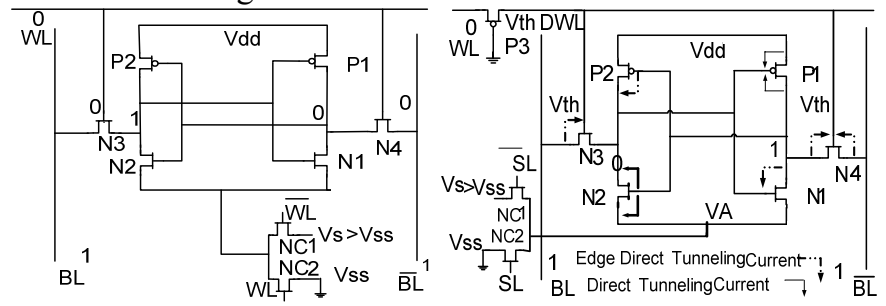

Fig. 1. (a) NC-SRAM [3]. (b) Proposed IWL-VC SRAM with gate leakage currents when the cell holds ' 0 .

\section{LOW GATE LEAKAGE SRAM CELLS}

In this section, we describe two low gate leakage SRAM cell structures, which are denoted as IWL-VC and PP-SRAM cells.

\section{A. IWL-VC SRAM Cell}

The initial configuration of the proposed SRAM cell is called WL-VC (Word Line-Voltage Control) SRAM cell [11]. In this cell, a pass transistor P3 (similar to $\mathrm{P} 3$ in

Fig. 1(b)) is added to the NC-SRAM cell to reduce the gate voltage of the N3/N4 pass transistors. This leads to the simultaneous reduction of both the gate tunneling and subthreshold currents in the idle mode. In the active mode, $\mathrm{WL}$ is ' 1 ', and the grounded-gate PMOS transistor P3 is ON, and hence, ' 1 ' is applied to the gates of transistors N3 and N4 while $V_{S S}$ is applied to the sources of transistors $\mathrm{N} 1$ and N2. Therefore, compared to the conventional SRAM, no change in the SRAM cell occurs. When the SRAM cell changes from the active mode to the idle mode, WL changes from ' 1 ' to ' 0 ' causing the source voltage of $\mathrm{P} 3$ to change from $V_{d d}$ in the active mode to a voltage higher than ' 0 ' (the PMOS threshold voltage, $V_{t h}$.) in the idle mode. This causes the gate voltages of $\mathrm{N} 3$ and $\mathrm{N} 4$ to increase to $V_{t h}$ as well. Furthermore, the sources of $\mathrm{N} 1$ and $\mathrm{N} 2$ are connected to $V_{S}$ through $\mathrm{NC1}$. Now, considering the case where a ' 0 ' is stored in the cell, $V_{\text {out }}$ increases to $V_{S}$ and DWL increases to $V_{\text {th }}$ (see

Fig. 1(b)). Thus, compared to the conventional SRAM cell, the absolute values of the gate-drain and the gate-source voltages of $\mathrm{N} 4$ and the gate-drain voltage of $\mathrm{N} 3$ decrease from $V_{d d}$ to $V_{d d}-V_{t h}$ while the gate-source voltage of $\mathrm{N} 3$ is $V_{t h}-V_{s}$. Consequently, the gate currents of transistors $\mathrm{N} 3$ and $\mathrm{N} 4$ is lowered. When the cell is storing ' 1 ', a similar gate current reduction is achieved.

To improve the timing performance of the WL-VC SRAM cell, instead of using WL and /WL in the WL-VC SRAM cell, we use SL and /SL signals to change the ground level sooner during the active mode.

Fig. 1(b) shows the new SRAM cell which is called the improved WL-VC SRAM (and is referred to as the IWL-VC SRAM from now on). In the read/write mode, WL (word line) and SL (select line) are high while in the idle mode, WL and SL are ' 0 '. In this configuration, the SL is always activated before $\mathrm{WL}$ is activated. This minimizes the degradations in the write and read operations compared to those of the conventional SRAM cell. In memories, several control signals, which are activated at different times, are used for the $\mathrm{read} /$ write operations. We shall use the first control signal during the operation for activating SL whereas WL is activated similar to the conventional SRAM cell [12]. To generate the first control signal, we use the signal Read and /W in the SRAM read and the write circuitry shown in Fig. 2 [13]. In the write mode when $/ \mathrm{W}$ is activated, the data is applied to the SRAM cell while in the read mode when Read is activated, the SRAM cell is ready for the read operation.

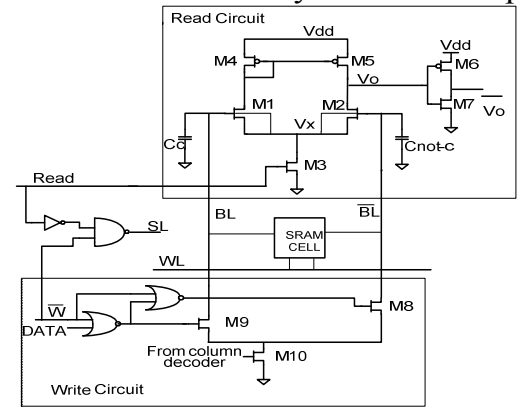

Fig. 2. The schematics of read and write circuits of the SRAM cell [13] and the additional logic for generating the SL signal.

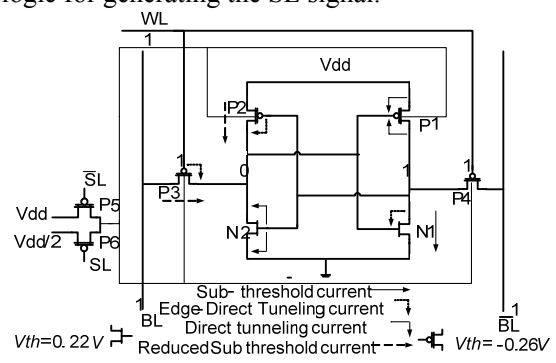

Fig. 3. Proposed PP-SRAM cell (holding ' 0 ') with gate leakage currents.

For both read and write operations, WL is activated after $/ \mathrm{W}$ and Read signals are activated, and hence, the size of $\mathrm{NC} 2$ need not be very large in order to prevent the speed degradation of the read and write operations. When SL becomes high, NC2 turns on taking $V_{A}$ (sources of N1 and N2) from $V_{S}(0.2 \mathrm{~V})$ to $V_{S S}(0)$. The WL signal is activated after $V_{A}$ has been stabilized at $V_{S S}$ (see subsection IV.D for the timing diagram). Since there is enough time for discharging the capacitance of the source line, the extra delay in the access time is avoided without resulting in a very large peak current. The static power consumption due to the two extra NMOS transistors ( $\mathrm{NC} 1$ and $\mathrm{NC} 2$ ) is not significant. In particular, the extra power dissipation for a row of 128 SRAM cells was estimated by circuit simulation to be less than $4 \%$ of the total static power dissipation.

\section{B. PP-SRAM CELL}

We present a gate leakage current reduction method based on PMOS Pass-transistor SRAM structure which is illustrated in 
Fig. 3. The PMOS Pass-transistor SRAM (called PP-SRAM) cell has lower gate leakage compared to that of the conventional SRAM cell. In order to decrease the gate leakage currents of the SRAM cell, NMOS transistors N3 and N4, are replaced by PMOS transistors $\mathrm{P} 3$ and $\mathrm{P} 4$. In the active mode, WL is held at ' 0 ' to turn on the two pass transistors. In the idle mode, WL is charged to $V_{d d}$ so that the two PMOS pass transistors are OFF, isolating the PP-SRAM cell from BL and /BL. During this time, the bit lines are typically charged to $V_{d d}$.

For this cell, the only gate leakage current of the pass transistor is $I_{G D 3}$, while in the conventional SRAM cell, three gate leakage currents i.e., $I_{G D 3}, I_{G D 4}$, and $I_{G S 4}$ are present. This fact alone leads to a considerable reduction in the gate leakage current of the SRAM cell. The use of PMOS pass transistor, however, may lead to performance degradation due to different mobility coefficients for the NMOS and PMOS transistors. To overcome this problem, the width of PMOS pass transistor is selected as 1.8 times of that of the NMOS for the technology used in this work. The ratio was obtained using HSPICE simulations for having the same transient characteristics for both types of transistors.

To decrease the subthreshold current in addition to the gate leakage current, the PMOS transistors with a higher threshold voltage may be used. In the proposed SRAM cell design, PMOS transistors with high threshold voltage $\left(V_{t h}=-0.26 \mathrm{~V}\right)$ and NMOS transistor with typical threshold voltage in $45 \mathrm{~nm}$ technology $\left(V_{t h}=0.22 \mathrm{~V}\right)$ are used [14] As observed from Fig. 3 , when ' 0 ' (' 1 ') data is stored, the subthreshold currents of P2 (P1) and P3 (P4) are reduced and the subthreshold current of N1 (N2) remains the same. In order to reduce the negative impact of high threshold voltage on the speed of the PPSRAM, a forward body biasing method is used. In this method, the body bias voltage of PMOS transistor in the idle mode is set to $V_{d d}$ (via P5) while in the active mode it is set to $V_{d d} / 2$ (via P6). The circuit diagram of the PP-SRAM cell with the body bias driver P5 and P6 is shown in Fig. 3.

Similar to IWL-VC SRAM cell, the select line (SL) signal is used to switch between the two body bias voltages. The signal is generated by the row decoder circuit. Note that the voltage of $V_{d d} / 2$ can be generated using an on-chip DC-DC converter or may be supplied externally. Since SL is activated before WL is activated for the read/write operation, the timing performance deterioration is prevented. It is important to point out that due to the use of the PMOS transistors, there is an increase in the dynamic power of the cell which is consumed during the read and write operation. Since static power is much more important than dynamic power in large memories, static power saving will very well compensate for the increase in dynamic power dissipation. In addition, the static power consumption induced by the two new inserted PMOS transistors (P5 and P6) is small. From circuit simulations, this component of power dissipation for a row of 128 SRAM cells was determined to be less than $2 \%$ of the total static power dissipation.

\section{RESULTS AND DISCUSSION}

To evaluate the efficiency of the proposed SRAM cells, we performed HSPICE circuit simulations for a $45 \mathrm{~nm}$ technology with the oxide thicknesses of $1.4 \mathrm{~nm}$ (the typical oxide thickness in the $45 \mathrm{~nm}$ technology is $1.4 \mathrm{~nm}$ ) [14]. In the simulations, temperatures of $25^{\circ} \mathrm{C}, 50^{\circ} \mathrm{C}$, and $100^{\circ} \mathrm{C}$ and the supply voltage of $V_{d d}=0.8 \mathrm{~V}$ were used. The channel widths of the main PMOS and NMOS transistors in the cell were $0.4 \mu \mathrm{m}$ and $0.2 \mu \mathrm{m}$, respectively.

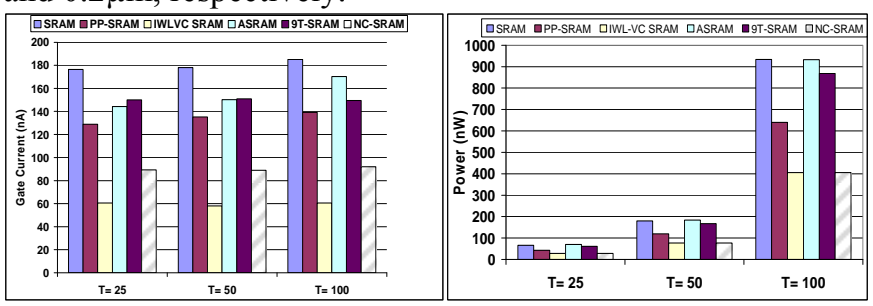

(a)

(b)

Fig. 4. (a) Gate currents and (b) power dissipations of SRAM cells at $25^{\circ} \mathrm{C}$, $50^{\circ} \mathrm{C}$, and $100^{\circ} \mathrm{C}$.

\section{A. Gate Leakage and Static Power Dissipation}

Simulation results of the gate leakage for all structures at the three temperatures of $25^{\circ} \mathrm{C}, 50^{\circ} \mathrm{C}$, and $100^{\circ} \mathrm{C}$ are presented in (a)

Fig. 4(a). As the results reveal, the leakage of the NC-SRAM cell, which makes use of two NMOS transistors for each row, is reduced by almost $50 \%$ compared to that of the conventional SRAM cell. The same reduction for the asymmetric SRAM cell of [6] is only about $20 \%$. In the first proposed structure, IWL-VC SRAM, with two NMOS and one PMOS transistors for each row, the gate leakage is reduced by about $66 \%$. For the 9T-SRAM cell of [9], which uses three more NMOS transistors, the gate leakage is lowered by $15 \%$. At last, in the PP-SRAM cell, by replacing the two NMOS pass transistors with PMOS pass transistors, the gate leakage current is reduced by almost $26 \%$. The maximum leakage reduction is achieved by the IWL-VC SRAM structure while the minimum reduction is seen in the asymmetric SRAM.

The total static power dissipations (included all leakage current components) for the SRAM cell structures are given in (a)

(b)

Fig. $4(\mathrm{~b})$ at $25^{\circ} \mathrm{C}, 50^{\circ} \mathrm{C}$, and $100^{\circ} \mathrm{C}$. As observed from the figure, compared to the conventional cell, only the asymmetric SRAM cell results in no static power saving. This can be attributed to the added NMOS transistor in this cell. For the 9T-SRAM cell, the static power reduction is smaller $(7.7 \%)$ because there are more transistors in the cell. The power dissipations in the NC-SRAM and the IWL-VC SRAM cell are similar and are $57 \%$ smaller than that of the conventional SRAM cell. Note that the power dissipations of added transistors in the NC-SRAM and IWL-VC SRAM cells are negligible. In PP-SRAM cell, use of the PMOS pass transistors and the high threshold PMOS transistors reduces the power dissipation by $37 \%$. As will be seen later, although the power dissipation and the leakage of the PP-SRAM cell are higher than those of the NC-SRAM and the IWL-VC SRAM cells, its read and write access times are the same as those of the conventional SRAM cell.

As known in the literature and seen in the figures, the gate leakage current does not depend on the temperature. The 
subthreshold leakage current, however, strongly depends on the temperature, making the total static power dissipation a strong function of the temperature. The power consumptions at different temperatures reveal that the same trends of power reduction for different cells exist at higher temperatures too. Finally, although the static power dissipation of the IWL-VC SRAM and NC SRAM cells are the same, the gate leakage of the IWL-VC SRAM is $34 \%$ less than that of the NC-SRAM mainly due to lower gate leakage currents of transistors N3 and N4. This is the main advantage of the IWL-VC SRAM compared to NC-SRAM. This gate leakage current decrease reduces the power dissipation of the row decoder due to the leakage current from the word line of each row which consists of many cells (say, from 32 to 512).

\section{Area}

The layouts of PP-SRAM and conventional SRAM cells drawn in a $45 \mathrm{~nm}$ standard CMOS technology are given in Fig. 5. Since the layout guidelines for the $45 \mathrm{~nm}$ technology was not available to us, we have used the layout guidelines presented in [15] which are a scaled version of the $90 \mathrm{~nm}$ technology. They could also be obtained by scaling the sizes and dimensions given in [16]. Because of the higher mobility of the NMOS transistors compared to that of the PMOS transistors, two PMOS pass transistors used in PP-SRAM cell are assigned larger widths. Thus, the area of the PP-SRAM cell is increased by $16.4 \%$ compared to that of the conventional SRAM cell. In the asymmetric SRAM cell, the addition of one extra transistor increases the area of the cell by $16.6 \%$ [6]. In the IWL-VC SRAM (NC-SRAM) cell, since only 3 (2) transistors are added per row, the increase in the area is negligible. For a row of 128 SRAM cells, the widths for $\mathrm{NC} 1, \mathrm{NC} 2$, and $\mathrm{P} 3$ were $4 \mu \mathrm{m}, 8 \mu \mathrm{m}$, and $6 \mu \mathrm{m}$, respectively, leading to a normalized area overhead of about $3 \%$. The NC-SRAM cell has the minimum increase in the area while the 9T-SRAM cell has the maximum area overhead [9].

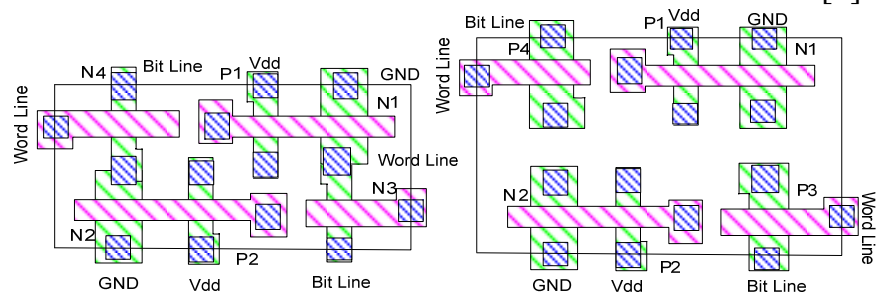

(a)

(b)

Fig. 5. Areas of (a) conv. SRAM, (b) PP-SRAM cells in 45nm technology.

\section{Read and Write Performances}

Now, we discuss the read and write performances of the SRAM cells. The timing diagrams in the read and write modes for the IWL-VC SRAM and PP-SRAM cells are depicted in Fig. 6 where the $/ W$ transition is considered as the timing reference (we have assumed an asynchronous SRAM.) There is a delay between the column address transition and the $/ W$ transition which is not included in our access time calculation. This delay, which is the same for all SRAM cells, only increases the total time, thereby decreasing the normalized increase in the access time (i.e., the ratio of the access time increase to the total access time.) Thus, the actual degradation percentage ought to be lower than the numbers reported here.

As the timing diagram for the IWL-VC write operation (Fig. 6 (a)) shows, about 200 ps after the $/ W$ transition (see Fig. 2) the $B L$ and $/ B L$ signals become stable in the selected SRAM cell. At this time, the $W L$ signal is activated to select the row and after $350 \mathrm{ps}$, the writing of the new data is finalized. This suggests that the cell has $200 \mathrm{ps}$ for restoring $V_{A}$ (see

Fig. 1(b)) to the actual ground. As shown in Fig. 6 (a), the transition of / $W$ induces a 0 to $V_{d d}$ transition of the $S L$ signal. When $S L$ is higher than the threshold voltage of NC2, this transistor turns on (see

Fig. 1(b)) taking $V_{A}$ to $V_{S S}$ well ahead of the $W L$ activation. Consequently, there is no access time increase due to the restoration of actual ground. Notice that, as shown in Fig. 6 (d), the amplitude reduction in $/ B L$ is less than $10 \%$ of the maximum swing, thus, no degradation in the sensing operation occurs due to the use of PMOS.

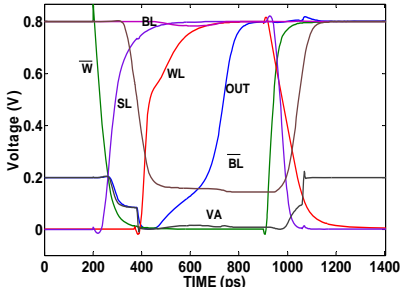

(a)

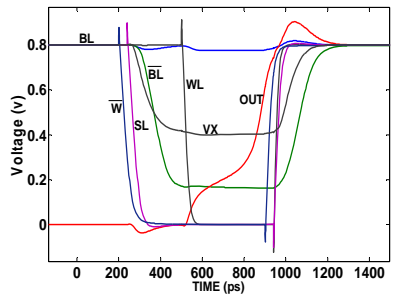

(c)

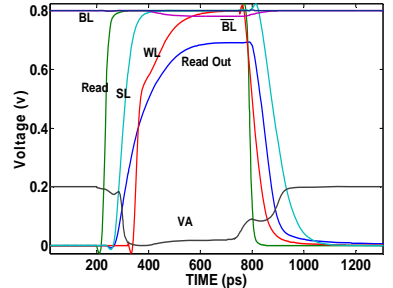

(b)

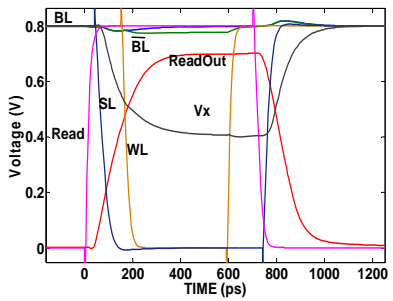

(d)
Fig. 6. Timing diagrams: (a) IWL-VC write, (b) IWL-VC SRAM read, (c) PPSRAM write, (d) PP-SRAM read operations.

Table I. READ AND WRITE DELAY INCREASES FOR EACH PROPOSED CELL COMPARED TO THOSE OF THE CONVENTIONAL SRAM CELL.

\begin{tabular}{|c|c|c|c|c|}
\hline Operation & A-SRAM & IWL-VC & PP-SRAM & NC-SRAM \\
\hline Write time & $4 \%$ & $4.4 \%$ & 0 & $4.3 \%$ \\
\hline Read time & $4 \%$ & $2.42 \%$ & 0 & $2.37 \%$ \\
\hline
\end{tabular}

The read and write delay increases for each proposed cell compared to those of the conventional cell are reported in Table I. Compared to the conventional SRAM cell, the performances of the read and write operations in NC-SRAM, IWL-VC SRAM, and asymmetric SRAM cells are degraded. In the NC-SRAM, because of using two NMOS transistors, through which a virtual ground is presented to the SRAM cell, the write and read access times are deteriorated by $4.3 \%$ and $2.37 \%$, respectively. In the IWL-VC SRAM, the read and write access times are degraded by $2.42 \%$ and $4.4 \%$, respectively. Although we activate the $S L$ signal sooner than in the case of NC-SRAM, the existence of the PMOS transistor (P3) which increases the resistance of the word line, degrades the access times. Without the added PMOS transistor, the read access time is degraded by $1.8 \%$ which is 
lower than what is observed in the IWL-VC SRAM cell. In addition, the $\mathrm{ON}$ resistance of $\mathrm{NC} 2$ increases the path resistance to the ground $\left(V_{S S}\right)$. The access time in the asymmetric SRAM cell is 4\% larger than that of the conventional SRAM cell. This degradation is due to the added N5 transistor. In the case of PP-SRAM cell, the timing performances remain unchanged. This originates from the fact that the forward body biasing technique is used for the PPSRAM cell read and write operations.

\section{E. Stability}

The stability of the SRAM cells may be determined by measuring the Static Noise Margin (SNM) value. Simulation results for the idle (retention) and read static noise margins of the proposed SRAM cells compared to the case of the conventional SRAM cell are reported in Table II. During the read mode, since the forward body biasing is used (the threshold voltage is decreased), the SNM is improved by $11 \%$. In the idle mode, the higher threshold voltage of the PMOS transistor, makes it more difficult for the access transistors to corrupt the data, thus yielding a 15\% higher SNM for this cell. In the IWL-VC SRAM cell, in the read mode, the presence of $\mathrm{NC} 2$ increases the path resistance to the ground $\left(V_{S S}\right)$ degrading the read SNM by $12 \%$. Also, in the idle mode, due to the virtual ground which is at a higher voltage than the actual ground, the SNM is degraded by $10 \%$. This is the price paid for lowering the static power consumption. Compared to the conventional SRAM, the stability of the asymmetric SRAM cells is slightly degraded [6], while the stability of 9T SRAM is improved by $100 \%$ [9].

Table II. SNM IMPROVEMENT OF THE PROPOSED CELLS COMPARED TO THAT OF THE CONVENTIONAL SRAM CELL.

\begin{tabular}{|c|c|c|c|c|}
\hline & IWL-VC SRAM & PP-SRAM & 9T-SRAM & ASRAM \\
\hline Retention Mode & $-10 \%$ & $+15 \%$ & 0 & negligible \\
\hline Read Mode & $-12 \%$ & $+11 \%$ & $100 \%$ & negligible \\
\hline
\end{tabular}

Since for the nanoscale SRAM, the $V_{t h}$ variation can be a serious problem, the $V_{t h}$ of the transistors in the SRAM cell is varied by $\pm \% 25$ to evaluate the effect of $V_{t h}$ on the SNM's. The effects of the $V_{t h}$ variations of transistors N1, N2, P1, P2, P3, and $\mathrm{P} 4$ in the PP-SRAM cell on the SNM's are reported in Fig. 7. As the results reveal, the SNM has a higher sensitivity to the $V_{t h}$ variations of P3 and P4. Since the structure of the SRAM cell does not change in the IWL-VC SRAM cell, the effect of the $V_{t h}$ variation on the SNM is similar to the SRAM cell presented in [17] and is not discussed. Note that since $\mathrm{NC} 1, \mathrm{NC} 2$, and P3 are not directly involved in the operations of the SRAM cell, their $V_{t h}$ variations do not directly influence the SNM's.

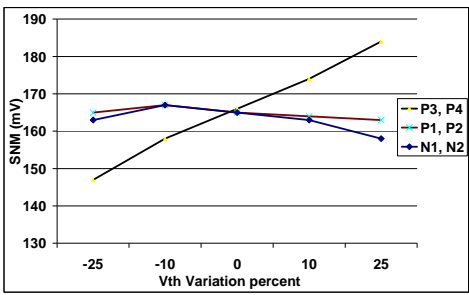

Fig. 7. Read SNM of the PP-SRAM cell as a function of $V_{t h}$ variation.

\section{CONCLUSION}

In this work, two new structures for the SRAM cell called IWL-VC SRAM and PP-SRAM were presented. The first cell structure made use of one PMOS per row of SRAM cells as well as two NMOS transistors for changing the ground voltage during the active and idle modes. This method lowered the gate current leakage of the cell by $58 \%$ and, hence, the static power dissipation of the memory with a minimum impact on the area. The read (write) access time of this SRAM was $4.4 \%$ $(2.42 \%)$ slower than that of the conventional SRAM. The static noise margin, however, was also lowered by $10 \%$ compared to conventional SRAM cell. In the second cell structure, PMOS pass transistors with high $V_{\text {th }}$ and forward biasing method were used to reduce both the gate oxide direct tunneling and the sub-threshold currents. In the PP-SRAM cell, the gate leakage current was reduced by $27 \%$ and the total static power by $37 \%$ while the read and write access times were not degraded. The SNM of PP-SRAM cell was also improved by $15 \%$.

\section{ACKNOWLEDGEMENT}

GR and AAK acknowledge the financial support by the Research Council of the University of Tehran.

\section{REFERENCES}

[1] 2007 International Technology Roadmap for Semiconductors, [online]. Available http://public.itrs.net.

[2] B. Yu et al., "Limits of gate oxide scaling in nano-transistors," in Proceedings of Symposium on VLSI Technology, pp. 90-91, 2000.

[3] P. Elakkumanan, C. Thondapu, and R. Sridhar, "A Gate Leakage Reduction Strategy for Sub-70nm Memory Circuit," in Proceedings of 2004 IEEE Dallas/CAS Workshop, 2004, pp. 145-148.

[4] J. Abraham, "Overcoming timing, power bottlenecks," EE Times, April 2003.

[5] K. M. Kao et al. "BSIM4 Gate Leakage Model Including Source-Drain Partition," in Proceedings of International Electron Devices Meeting, pp. 815-818, Dec 2000.

[6] N. Azizi and F.N. Najm, "An Asymmetric SRAM cell to lower gate leakage," in Proceedings of 5th IEEE International Symposium on Quality Electronic Design, 2004, pp. 534-539.

[7] S. Yang et. al., "Low-Leakage Robust SRAM Cell Design for Sub100nm Technologies," in Proceedings of Asia South Pacific Design Automation Conference 2005. pp. 539-544.

[8] B. Amelifard, F. Fallah, and M. Pedram, "Reducing the sub-threshold and gate-tunneling leakage of SRAM cells using dual-Vt and dual-Tox assignment," in Proceedings of DATE, pp. 1-6, March 2006.

[9] Z. Liu and V. Kursun, "Characterization of a Novel Nine-Transistor SRAM Cell," IEEE transactions on VLSI Systems, vol. 16, no. 4, pp. 488-492, April 2008.

[10] K. Kim, H. Mahmoodi, and K. Roy, "A Low-Power SRAM Using BitLine Charge-Recycling," in the Proceedings of International Symposium on Low-Power Electronics Design, August 27-29, 2007, pp. 177-182.

[11] G. Razavipour, A. Motamedi, and A. Afzali-Kusha, "WL-VC SRAM: A Low Leakage Memory Circuit for Deep Sub-Micron Design," in Proceedings of 2005 IEEE International Symposium on Circuits and Systems, Island of Kos, Greece, May 21-24, 2006, pp. 2237-2240.

[12] C.H. Kim, J. Kim, S. Mukhopadhyay, and K. Roy, "A Forward BodyBiased Low-Leakage SRAM Cache: Device, Circuit and Architecture Considerations," IEEE Transactions on Very Large Scale Integration (VLSI) Systems, vol. 13, no. 3, pp. 349-357, March 2005.

[13] J. M. Rabaey, A. Chandrakasan, and B. Nikolic, Digital Integrated Circuits, Prentice-Hall, 2003.

[14] Predictive Technology Modeling, http://www.eas.asu.edu/ ptm.

[15] F. Arnaud et. al., "A Functional $0.69 \mu \mathrm{m}^{2}$ Embedded 6T-SRAM bit cell for $65 \mathrm{~nm}$ CMOS platform," in the Digest of Technical Papers of the Symposium on VLSI Technology, 10-12 June 2003, pp. 65-66. 
[16] Zheng Guo et. al. "FinFET-based SRAM design," in the Proceedings of the International Symposium on Low Power Electronics and Design, 810 Aug. 2005, pp 2-7.

[17] B. H. Calhoun and A. Chandrakasan, "Analyzing Static Noise Margin for Subthreshold SRAM in 65nm CMOS," in Proceedings of ESSCIRC, pp. 363-366, France, 2005. 